\title{
Iterative Didactic Design of Serious Games
}

\author{
Michael G. Wagner \\ Drexel University \\ 3141 Chestnut Street 71-220C \\ Philadelphia, PA 19104, USA \\ michael.g.wagner@drexel.edu
}

\author{
Thomas Wernbacher \\ Danube University Krems \\ Dr.-Karl-Dorrek-Straße 30 \\ 3500 Krems, Austria \\ thomas.wernbacher@donau-uni.ac.at
}

\begin{abstract}
In this paper we present a novel approach for the development of serious games that integrates elements from instructional design with the iterative design methodology used in commercial game development. Our approach is motivated through a theoretical model of game based learning that borrows key concepts from Heinz Von Foerster's epistemology of radical constructivism and James Paul Gee's three-identity model. We furthermore detail how our approach was used in the development of Ludwig, an award winning physics learning game for middle school classroom use. An evaluation of how our method proved successful in the development of Ludwig along with an outlook on future research concludes this paper.
\end{abstract}

\section{Categories and Subject Descriptors}

K.8.0 [Personal Computing]: General - Games. D.2.9 [Software

Engineering]: Management - Software process models.

\section{General Terms}

Management, Design, Human Factors, Theory.

\section{Keywords}

Serious Games, Game Based Learning, Game Design, Game Studies.

\section{INTRODUCTION}

Our current view on educational games as well as serious games in general is mainly driven by the idea that they provide a powerful tool for learning and personal advancements [1, 2]. At the same time current research also indicates that the transfer potential of games seems much less powerful when it comes to unwanted and socially dangerous concepts or emotions such as violence or aggression [10]. It is often overlooked that there appears to be an inconsistency in research results indicating that the transfer mechanism found in games are not yet sufficiently understood.

In this paper we want to address this problem through the development of a theoretical concept that is capable of explaining this apparent inconsistency. At the same time we will utilize this concept in order to deduce a design approach that is capable of optimizing the usability of a game for a serious purpose during its development process.

\section{LUDIC CONSTRUCTIVISM}

We start by outlining a theoretical approach we developed in order to explain the knowledge transfer mechanisms specific to digital games. Our theory is built on James Paul Gee's threeidentity model [7] and largely follows an argument first made by Heinz von Foerster in his radical constructivist epistemology [17]. It should be noted that this theory requires us to focus on complex games [14], i.e. games that support a virtual player presence, and that the predictions made by our theory are consistent with the results of a series of empirical studies we conducted on the use of computer games in schools [11].

According to Gee a player in a complex video game will develop three identities consisting of a virtual and a real identity situated within and outside the game space, respectively, as well as a so called projecting identity responsible for establishing a connection between the virtual and real identities (cf. Figure 1).

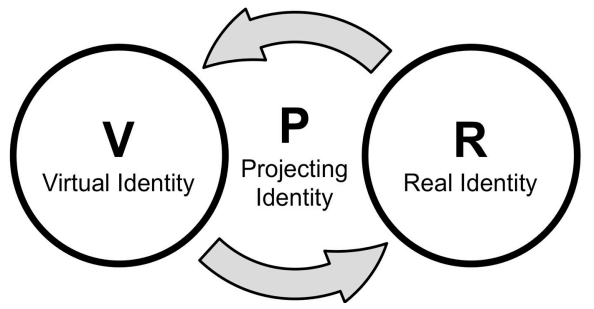

Figure 1. Gee's three identity model

For the following it is useful to think of the projecting identity in terms of two directions, the forward-projecting identity $P_{f}$ that is responsible for mapping the real onto the virtual identity and the back-projecting identity $\mathrm{P}_{\mathrm{b}}$ for mapping the virtual onto the real identity [20]. In a complex game, a player's virtual identity can then be seen as a function of the real identity

$$
\mathrm{V}=\mathrm{P}_{\mathrm{f}}(\mathrm{R}) \text {, }
$$

and vice versa his or her real identity as a function of his or her virtual identity

$$
\mathrm{R}=\mathrm{P}_{\mathrm{b}}(\mathrm{V}) \text {. }
$$

When combined into one equation, these dependencies describe how singular game play instance would influence the real identity of a player

$$
R^{*}=P_{b}(V)=P_{b}\left(P_{f}(R)\right),
$$

or, in a simplified notation

$$
R^{*}=G(R) \text {, with } G=G\left(P_{f}, P_{b}\right) .
$$

As Gee pointed out, the projecting identity constantly negotiates between virtual and real identities during game play [7]. Above 
equation therefore cannot be seen as a simple function that describes how a game influences our real identity. Rather, it describes a recursion in which each change in the real identity is immediately fed back into the system. Consequently, we get

$$
R^{n+1}=P_{b}\left(P_{f}\left(R^{n}\right)\right)=G\left(R^{n}\right)=G\left(G\left(R^{n-1}\right)\right)=\ldots
$$

It is important to note that a single recursion in this description is not to be confused with a single game play interaction. Rather, it describes one miniscule entity of influence of the player's real identity during the process of game play. For detailed information on how an analogous argument has been used in studying our general understanding of understanding, we refer the reader to von Foerster's work on the epistemology of radical constructivism and second order cybernetics [19].

If the underlying game system has any consistent influence on the player's real identity, the recursion will ultimately stabilize as described by

$$
R^{\infty}=P_{b}\left(P_{f}\left(R^{\infty}\right)\right)=G\left(R^{\infty}\right) .
$$

Values $x$ that solve an equation of the form $x=f(x)$ are called the eigenvalues of $f$. The recursion describing how a game influences the real identity of the player through transfer processes will therefore stabilize at an eigenvalue of $G=G\left(P_{f}, P_{b}\right)$. Since this function in return exclusively depends on the two directions of the projecting identity we finally arrive at the following conclusion:

On the long run, a complex video game influences a player in such a way that the resulting real player identity represents an eigenvalue of the projecting player identity.

This seemingly simple statement has far reaching consequences on our understanding or games and their transfer effects. In this paper we will focus on how this influences our understanding of serious games and how we can utilize this knowledge in serious games development through the concept of iterative didactic design. The general consequences of this theory will be subject of a subsequent paper on ludic constructivism [23].

\section{LIMITATIONS OF SERIOUS GAMES}

Eigenvalues are fundamentally nonlinear and thus exhibit what is commonly referred to as the butterfly effect. Even the smallest changes in $G\left(P_{f}, P_{b}\right)$ can have drastic effects on the resulting Eigenvalues of $G\left(P_{f}, P_{b}\right)$. In addition, we have seen that $G\left(P_{f}, P_{b}\right)$ depends not only on the game itself, but more importantly on the real and projecting identities of the player. The projecting identity in return also depends on the environment in which the game is played in. This causes significant limitations on the potential of using games for serious purposes.

In fact, it is not so much the game itself but rather the way a game is played or used that is responsible for transfer effects to occur. In this sense, being serious can never exclusively be a property of a game as a player independent system but only a property of the game's usage within a serious and player centered context. Every player of every game learns something from playing the game but what he or she learns depends mainly on the player's personality as well as the environment in which the game is played. Any game can therefore be used as a serious game and in return any game currently referred to as a serious game can be played without its serious purpose and without any observable transfer effect supporting that purpose.

We have been able to verify this fundamental result through a series of empirical tests conducted over a period of 8 years funded by the Austrian Federal Ministry of Education [11]. In this project we introduced the use of off the shelf computer games into regular classroom activities in various schools in Austria and studied their pedagogical effects. We particularly investigated how the way teachers and students used the games in classroom influenced the educational potential of this didactic approach. The final results of this project have been published as a handbook on the use of computer games in the classroom that is freely accessible to all Austrian teachers through the Austrian Federal Ministry of Education [22].

In short, the results we observed can be summarized in three key statements, which outline the potential as well as the limitations of computer games in formal education.

1. Digital games do not teach, teachers do. Our studies clearly show that teachers are of essential importance in digital game based education. The current emphasis on technology that arose out of the possibilities provided by new media tend to cloud the fact that even with the enhanced potential, the medium always remains a medium. Classroom learning, in particular at the elementary and middle school levels, is driven by the interaction between the teacher and the students.

2. Teachers who want to use digital games in the classroom do not need to be gamers. The main role of a teacher in digital game base education within a formal setting is to make sure that the recursive process described by $G\left(P_{f}, P_{b}\right)$ leads to outcomes that correspond to the curriculum. These outcomes are supposed to be equal or at least comparable for all students in the classroom. The teacher hence takes over the role of a process manager that ensures, through continuous pedagogical intervention, that the learning processes of all students are synchronized. In our project teachers who were able to assume this role were more successful in using digital games even if they had no prior experience with games themselves.

3. Students who play digital games in their spare time get more out of digital games based education. It turns out to be a myth that all students that grew up with digital media are also what Prensky called digital natives [13]. In fact, we could show that a significant number of students had no interest in digital media or computer games even though they had access to the technology as well as experience in using it. This is not really surprising considering that people tend to have varying media preferences and digital games are just another medium in the mix. In our project we found that approximately 20 percent of the students showed little or no interest in digital games and that these students generally did not benefit from the use of games in the classroom [11].

It should be noted that these limitations also have important positive consequences as they limit unwanted effects such as the transfer of aggression and violence as well. As with regular learning, the transfer of aggression requires that the player's real and projecting identities are susceptible to such an emotional state. And while it is possible that either the player personality or the environment in which a game is played support transfer of negative and unwanted emotions, it is highly unlikely to occur within the regular gameplay of a mentally healthy player in a socially stable environment.

While this underlines our argument that it is not the game system but rather the game usage that can make a game serious, the term serious game is not completely without merit. In fact, even though any game can be used seriously, there are games that are easier to apply in serious use cases than other games. It is important to remember, however, that it is not the game itself; it is much more 
the use of the game that has a serious purpose. Serious game design therefore has to be thought of not as the design of games with a serious purpose but rather as the design of games that support applications within contexts with serious purpose. This is an important distinction.

In the following chapters we will outline the principles of this approach to serious game design by examining the development of the physics leaning game Ludwig. Before we can get into detail on the actual game, however, we first need to look at how the standard processes currently applied in game design and instructional design are fundamentally different and how we can combine them into one development approach suitable for our interpretation of serious game design.

\section{ITERATIVE DIDACTIC DESIGN}

Even though some recent models employed in instructional design include limited iterative concepts and some ideas originating in agile software development such as rapid prototyping [8], current instructional design methodologies in general follow processes that have their roots in the so-called ADDIE (Analyze, Design, Develop, Implement, Evaluate) model [15]. In this approach, the design process relies on the a-priori definition of the desired learning outcomes along with a pre-set methodology on how these outcomes will be achieved through instruction. Even if a design model includes iterative feedback loops, it is always restricted in terms of the actual instructional outcome and methodology it aims for.

In contrast to this approach, game design follows a much more radical path. In game design the initial development goal is a playable prototype that can be tested immediately. Test results obtained from so-called playtesting are then analyzed and fed back into the design process where they can influence any part of the game. A new prototype based on the adjusted requirements is built and tested again leading to a rapid recursive design process commonly referred to as iterative design [6] (cf. Figure 2). The important aspect in this process is the fact that in this case any part of the game is subject to evaluation and potential change leading to a much less restricted development process that might lead to a game that deviates from the original game concept substantially.

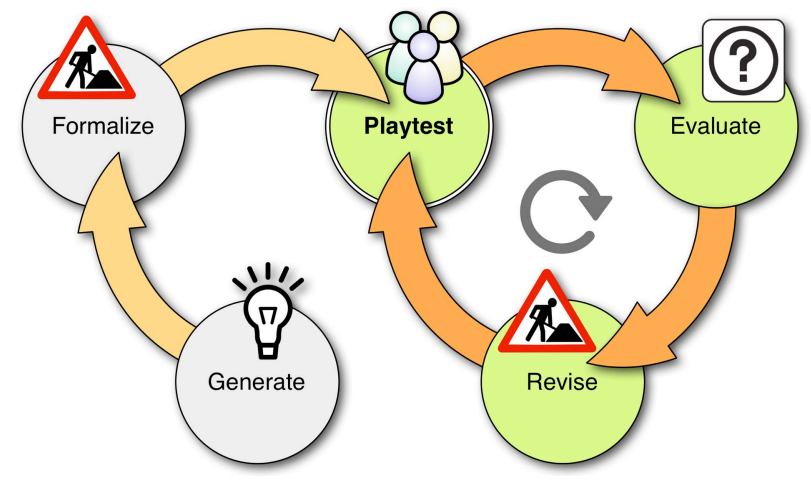

Figure 2. Iterative design model

As with any recursive model, iterative design creates a process that seeks to heuristically optimize the parameters that are subject to change within a single iteration. In other words, the set of playtesting questions as well as the playtesters themselves become the driving factors for the development of the game. If, for example, the main emphasis of the playtesting phase is to evaluate player experience within a group of male adolescent playtesters, the corresponding iterative design process will tend to evolve the prototype into a game that optimizes player experience of adolescent males. In this sense the successful creation of emotions such as fun or suspense within a game is as much the result of the actual development process as it is a consequence of the overall game concept by a game designer or game design team.

Educational games in particular as well as serious games in general are usually developed with the use of instructional design methodologies in order to ensure that the requirements for their specific learning outcomes or serious goals are met. Since the evolutionary development effect of optimizing fun and intrinsic motivation through continuous playtesting is generally absent in these design models, creating fun in serious games constitutes a significant challenge. In addition, instructional design does not account for the fact that learning in games is dependent on the personalities of the players and the learning situations in which the games are played.

Through the specification of the playtesting process, including the selection of the playtesters as well as the situation in which the playtesting takes place, iterative design is capable to custom design a game for a particular target group and application scenario. It is therefore possible to setup the design process in such a way that it heuristically optimizes the recursive process described by $G\left(P_{f}, P_{b}\right)$ in a direction that supports predefined learning outcomes within a certain educational setting. For this purpose, we have proposed to enhance the traditional playtesting methodology with an educational evaluation that is performed in as many design iteration as possible [24]. In the following we briefly outline a game that was developed using our iterative didactic design process.

\section{LUDWIG}

Ludwig is an interactive 3D-adventure game built around the topic of renewable energy production [12] and aimed for use as an in-class learning tool in middle school physics. Players are allowed to explore the game world freely without any limitations set by the instructional or game design. While we emphasized pedagogical developments with respect to educational standards for physics, the heart of our project lies in its iterative game design. Ludwig was utilized and optimized gradually based on the feedbacks of participatory students and teachers. Usability, fun of play, motivational factors and learning progress were investigated during workshops, which took place at the location of the developer ovos in Vienna as well as schools all around Austria. At these workshops, cognitive transfer effects (interest, self-efficacy) were measured in a summative evaluation and the results of these measurements were fed back into the development process.

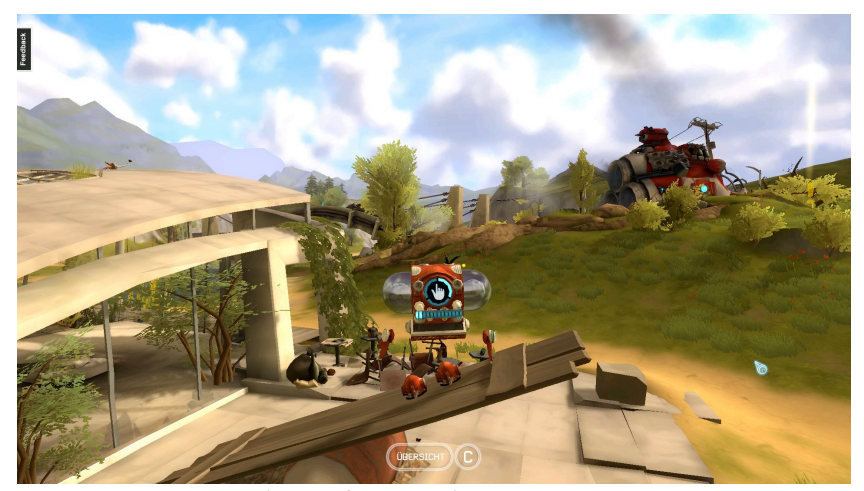

Figure 3. Ludwig screenshot 
One of our main goals was to develop a learning game that could be compared to video games our target group has become accustomed to. A significant amount of effort has therefore been put into creating a lush 3D game world with a high amount of interactive objects (cf. Figure 3).

The learning progress in our game is mapped by knowledge points, which are received when important quests are solved or if the player finds certain resources. The more he or she explores the virtual world the more content is unlocked and added to a knowledge base describing important facts related to energy such as combustion or wind energy (cf. Figure 4). In order to allow the game to be used as an in-class teaching tool, these facts are closely aligned with the Austrian physics curriculum. With respect to the design of our knowledge base, usability criteria based on national DIN norms were taken into consideration as well.

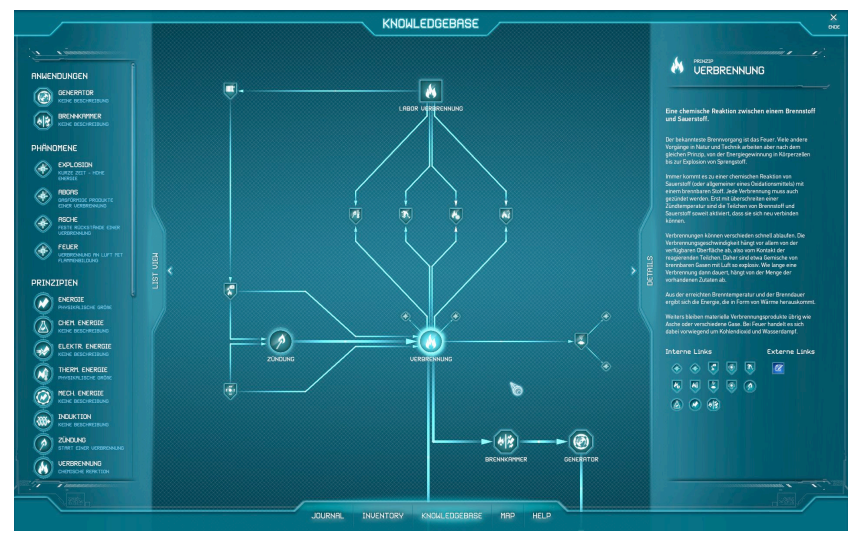

Figure 4. Knowledge base

Ludwig focuses intrinsic motivation [16] by implementing the learning goal deeply into the game so that the learning goal becomes as closely connected to the game objective as possible. In addition it offers a virtual laboratory for the learner (cf. Figure 5). This laboratory can be seen as "game within the game" in which players utilize resources the collected in the game such as coal, wood or paper. The laboratory simulates physical models in an experimental context that can also be recreated in real life within the classroom. By using this approach, science is made accessible in game as well as in real life. It is important, however, to support autonomous learning methods by giving the player full control over his actions without implementing any time limits or sequential arrangements.

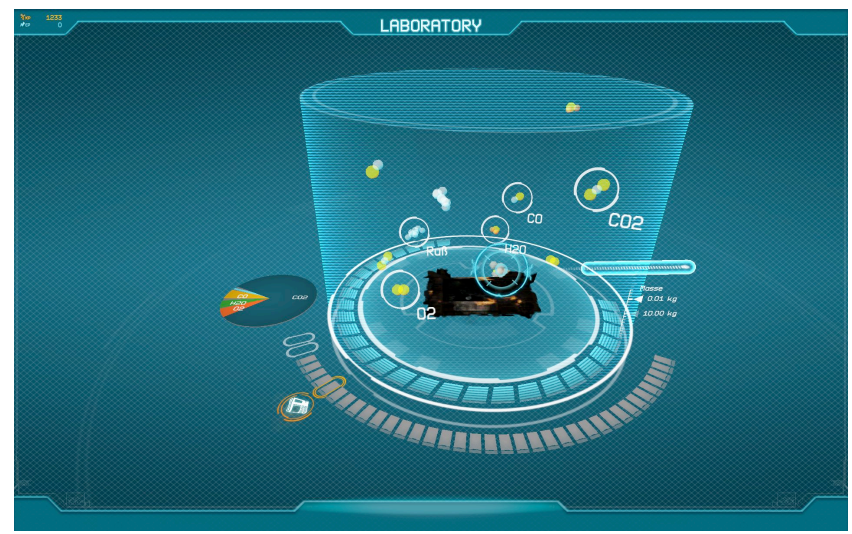

Figure 5. Virtual laboratory
From a constructivist point of view, learning processes depend on the grade of involvement in the respective activity. While solving contextualized tasks student's skills are developed by working on the problem through authentic activities [5]. The entry level of Ludwig itself provides plenty of opportunities for learners to work immediately on realistic problems like starting a fire by means of combining resources found in the game world. Later levels deal with water, wind and solar energy.

\section{DIDACTIC PLAYTESTING}

For the didactic playtesting we applied quantitative methods in order to precisely operationalize values of a particular variable (answers in questionnaires) for further statistical computations, while also using qualitative methods, which allow for a systematic interpretation of the meaning of verbal material (interviews, workshops). We decided to combine elements from both approaches in order to get a holistic view on the acceptance and effects of our game.

The process relied on feedback from students as well as teachers. By means of this feedback we aimed at identifying problems and enhancing the overall quality of Ludwig iteratively during the entire development phase. In order to assess the overall fun factor and playability we adapted quality criteria developed by the "Federal Office for the Positive Assessment of Computer and Console Games" (BUPP) [4], which is a service agency of the Austrian Ministry of Economy, Family and Youth. This institution offers guidance for parents and kids by rating video game titles and hosting a review database on this site. These criteria describe the following key aspects of gameplay: audiovisual quality, controls, fun of play, level of difficulty, performance, playability $\&$ suitability. Through this user based design process we were able to enhance the playability of the learning game. Practical issues relating to the school context (technical resources, lack of it-rooms) were likewise considered by accounting for the teacher's perspective.

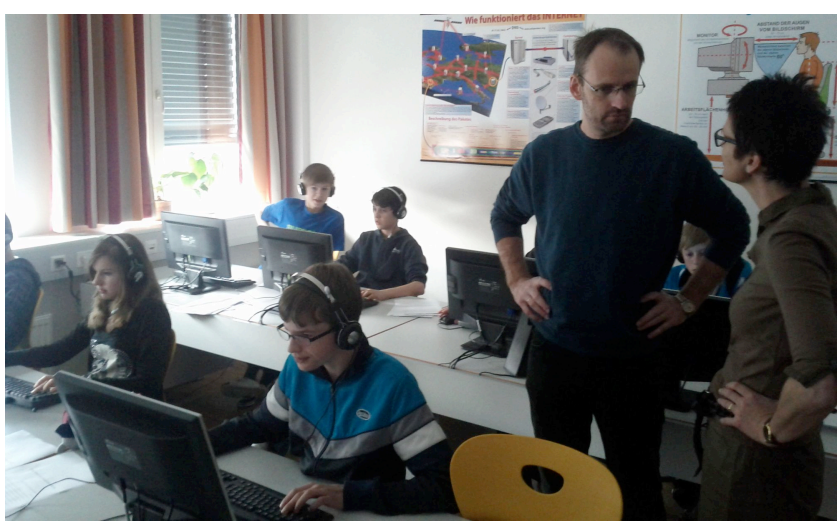

Figure 6. Playtesting session

Ten school classes took part in the iterative didactic design process of Ludwig by providing their individual view of the game during play-testing sessions. Between spring of 2011 and 2012, four main iterations were conducted that included semi structured interviews with teachers on the acceptance and application of learning games in class. Students from different high schools received questionnaires referring to the usability of the game (interface, control of avatar), to the playing experience (fun of play, immersion), to motivational factors (intrinsic motivation, self-efficacy, interest for physics) as well as to knowledge gains. For measuring self-efficacy and interest we used questionnaires 
from the Pisa studies (PISA assesses to what extent students at different educational levels have acquired essential knowledge and skills). For measuring intrinsic motivation we utilized a questionnaire developed by the Austrian Knowledge Management Institute [3]. Fun of play, perceived level of difficulty and immersion were determined using a questionnaire on game experience by Klimmt [9].

One of the main challenges in this approach is the number of iterations that can be performed during game development. As it is impossible to "unlearn" content that has been learned as part of a playtesting session, each process iteration had to be conducted by a different playtesting group. While it is of key importance to have as many development iterations with integrated didactic playtesting as possible, we found that four iterations were sufficient to guide the development process towards a game that could address the required learning goals. In addition, traditional playtesting focusing in playability and overall usability was performed at a significantly faster iteration rate in line with the standards used in commercial game development.

\section{EVALUATION}

In order to analyze the success of our approach, we conducted a summative evaluation in which we focused on the quality and the impact of the final version of the first game level of Ludwig. Our goal was to measure possible knowledge transfer effects, changes in interest for physics and changes in student's self-efficacy concerning physics. To put it in other terms, we wanted to know if Ludwig alters motivational and cognitive factors: can we pick up students at their actual individual knowledge and motivational states and get them to the next level in a playful way?

Four classes from two different schools totaling eight teachers and 80 students participated in this process. In order to evaluate transfer effects, we followed a $2 \times 2$ pre-post design meaning that student's knowledge concerning the subject physics, perceived instructional quality, self-efficacy and student's interest for physics were investigated at the beginning and the end of term. In our test design, transfer effects regarding knowledge, interest and self-efficacy were defined as dependent variables. Testing time (beginning/end of term) and grade of feedback by the teacher (discussion of game content yes/no) were defined as independent variables. Game experience and sex were used as mediator variables.

Through this evaluation along with the analysis provided by the individual playtesting evaluations we were able to prove that Ludwig did lead to knowledge transfer as long as the playing sessions are well reflected by teachers and students and that the didactic design process had a positive effect on the educational potential of the game [23]. In addition, we could demonstrate that the game content has to be linked to existing physical problems in order to facilitate real world learning not restricted to the virtual world of the game itself. These results strongly support the theoretical approach outlined in this paper.

We furthermore interviewed teachers that took part in our playtesting sessions with respect to the opportunities and challenges of using serious games in general and Ludwig in particular as part of regular in-class activities. According to all involved teachers Ludwig was able to trigger self-reliant learning strategies, to enhance student's motivation concerning physics and to make students curious about physical principles. Potential obstacles that stand in the way of a broad application of serious games in educational settings were identified as tight schedules, poor IT infrastructure at schools and lacking resources for preparation [23].

\section{CONCLUSION}

In this paper we used a theoretical approach borrowed from second order cybernetic theory in order to explain the potential as well as the limits of serious games and utilized this approach as a basis for an iterative didactic design process capable of optimizing the educational usability of games. We furthermore explained how we used iterative didactic design in the development of the physics learning game Ludwig and how we were able to demonstrate the success of our approach through a quantitative as well as qualitative evaluation.

While iterative didactic playtesting has proven to be an excellent tool for optimizing games for serious application scenarios within the development phase, there is still significant potential for future improvements and research. In particular, we relied on standardized tests in measuring self-efficacy and intrinsic motivation. This was needed in order to ensure that our approach could be validated properly from a research perspective. However, since game development in general does not require such a high level of research accuracy, we feel that the tests used for iterative didactic playtesting could be reduced in complexity in order to make the process more manageable from a game development perspective.

A larger research question arises from the fact that our research suggests that the formal educational use of games requires a significant amount of learning process management through a teacher or trainer. This contradicts opinions that games are excellent tools for self-directed learning and would indicated that it is difficult if not impossible to achieve economies of scale in educational game development. One way to approach this problem would be to implement intelligent tutoring systems at the meta-level of the game so that the pedagogical structure of the game is capable of adjusting itself autonomously based on analyzing the projecting identity of the player. This, however, would not only call for significant advancements in the automated understanding of individual player personalities, it would also require additional research in automated storytelling by means of artificial intelligence.

\section{ACKNOWLEDGEMENTS}

Ludwig was produced by Vienna based ovos realtime3d $\mathrm{GmbH}$ through funds provided by the Austrian Federal Ministry of Science and Research, the Austrian Federal Ministry of Education, Arts and Culture, the City of Vienna, VERBUND Austria and the Austrina Climate and Energy Fund. The evaluation of Ludwig was supported through the Sparkling Science program of the Austrian Federal Ministry of Science and Research and the Austrian Federal Ministry of Education, Arts and Culture.

Since its release in 2011 Ludwig has received a number of international awards, including the German Game Developer Award for best serious game 2011, the e-Virtuoses award 2012 for best game in education and training as well as the Worlddidac Award 2012. The game has furthermore received positive reviews from the gaming press [18]. 


\section{REFERENCES}

[1] Annetta, L.A. 2010. The "I's" have it: A framework for serious educational game design, Review of General Psychology, 14 (2), pp 105-112.

[2] Annetta, L. A., Cook, M. P., and Schultz, M. 2007. Video games and universal design: A vehicle for problem-based learning, Journal of Instructional Science and Technology, $10(1)$.

[3] Bedek, M.A., Seitlinger, P., Kopeinik, S. and Albert, D. 2011. Multivariate Assessment of Motivation and Emotion in Digital Educational Games, in Gouscos, D. and Meimaris, M. (eds.) Proceedings of the 5th European Conference on Game-Based Learning, Athens, Greece, October 20-21, 2011, pp. 18-25.

[4] Bundesministerium für Wirtschaft, Familie und Jugend 2012. BUPP. Bundesstelle für Positivprädikatisierung von Computer- und Konsolenspielen. http://www.bupp.at.

[5] Duffy, Th.M., and Cunningham, D. J. 1996. Constructivism: implications for the design and delivery of instruction, in Jonassen, D.H. (ed.), Handbook of Research for Educational Communications and Technology New York: Macmillan, pp. 170-198.

[6] Fullerton, T. 2008. Game Design Workshop: A Playcentric Approach to Creating Innovative Games. Mcgraw-Hill.

[7] Gee, J. 2007. What Video Games Have to Teach Us About Learning and Literacy. Second Edition: Revised and Updated Edition. Macmillan.

[8] Hokanson, B., and Miller, C. 2009. Role-based design: A contemporary framework for innovation and creativity in instructional design. Educational Technology 49(2), pp. 2128.

[9] Klimmt, Ch., Hefner, D., Vorderer, P. and Roth, Ch. 2008. Exploring the Complex Relationships Between Player Performance, Self-Esteem Processes, and Video Game Enjoyment. Paper presented at the annual meeting of the International Communication Association, Montreal, Quebec, Canada, May 21, 2008.

[10] Kutner, L. and Olson, Ch. 2008. Grand Theft Childhood: The Surprising Truth About Violent Video Games and What Parents Can Do. Simon and Schuster.
[11] Mitgutsch, K, and Wagner, M.G. 2009. Gaming the Schools: Didaktische Szenarien des Digital Game Based Learning, Medienimpulse 2/2009.

[12] Ovos realtime3D GmbH 2012. Luwdig. Meet Ludwig. http://www.playludwig.com.

[13] Prensky, M. 2001. Digital Natives, Digital Immigrants. On the Horizon, 9(5), pp. 1-6.

[14] Prensky, M. 2005. In Educational Games, Complexity Matters. Mini-games are Trivial - but "Complex" Games Are Not, Educational Technology 45(4), pp. 1-15.

[15] Reiser, R. A. and Dempsey, J. V. 2012. Trends and issues in instructional design and technology. Pearson.

[16] Ryan, R. M. and Deci, E. L. 2000. Self-determination theory and the facilitation of intrinsic motivation, social development, and well-being, American Psychologist 55, pp. 68-78.

[17] Segal, L. 1986. The Dream of Reality: Heinz Von Foerster's Constructivism. W W Norton \& Co Inc.

[18] Taylor, D. 2011. Play Ludwig teaches students physics in a brilliant, interactive game, The Next Web. http://thenextweb.com/eu/2011/12/06/play-ludwig-teachesstudents-physics-in-a-brilliant-interactive-game/

[19] Von Foerster, H. 2002. Understanding Understanding: Essays on Cybernetics and Cognition. Springer.

[20] Wagner, M.G. 2007. Identitätsrückprojektion in Aktiven Medien, e-beratungsjournal.net, Fachzeitschrift für OnlineBeratung und computervermittelte Kommunikation 3(2).

[21] Wagner, M.G. 2008. Serious Games: Spielerische Lernumgebungen und deren Design, in: Issing, L.J. and Klimsa, P. (eds.), Online-Lernen - Handbuch für Wissenschaft und Praxis, Oldenburg Wissenschaftsverlag, pp. 297-306.

[22] Wagner, M.G., and Gabriel, S. 2011. Didaktische Szenarien des Digital Game Based Learning, Handreichung für Lehrkräfte, bm:ukk.

[23] Wagner, M.G. 2013. Ludic Constructivism, under review.

[24] Wernbacher, T., Pfeiffer, A., Wagner M.G. and Hofstätter, J. 2012. Learning by Playing: Can Serious Games be fun?, in: Proceedings of the 6th European Conference on Games Based Learning (ECGBL 2012), Cork, Ireland, October 4-5, 2012, pp. 533-541. 